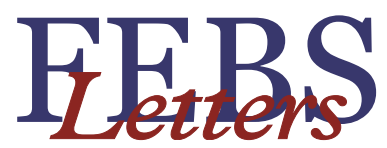

journal homepage: www.FEBSLetters.org

\title{
The mode of $\alpha$-synuclein binding to membranes depends on lipid composition and lipid to protein ratio
}

\author{
Volodymyr V. Shvadchak $^{\mathrm{a}, *}$, Dmytro A. Yushchenko ${ }^{\mathrm{a}, 1}$, Roberta Pievo ${ }^{\mathrm{b}}$, Thomas M. Jovin ${ }^{\mathrm{a}}$ \\ a Laboratory of Cellular Dynamics, Max Planck Institute for Biophysical Chemistry, am Faßberg 11, 37077 Göttingen, Germany \\ ${ }^{\mathrm{b}}$ Electron Paramagnetic Resonance Group, Max Planck Institute for Biophysical Chemistry, am Faßberg 11, 37077 Göttingen, Germany
}

\section{A R T I C L E I N F O}

\section{Article history:}

Received 12 August 2011

Revised 1 October 2011

Accepted 3 October 2011

Available online 12 October 2011

Edited by Sandro Sonnino

\section{Keywords:}

Environment-sensitive dye

Fluorescence

ESIPT

Protein conformation

EPR

\begin{abstract}
A B S T R A C T
Interactions of the presynaptic protein $\alpha$-synuclein with membranes are involved in its physiological action as well as in the pathological misfolding and aggregation related to Parkinsons's disease. We studied the conformation and orientation of $\alpha$-synuclein bound to model vesicular membranes using multiparametric response polarity-sensitive fluorescent probes together with CD and EPR measurements. At low lipid to $\alpha$-synuclein ratio the protein binds membranes through its $N$-terminal domain. When lipids are in excess, the $\alpha$-helical content and the role of the C-terminus in binding increase. Highly rigid membranes also induce a greater $\alpha$-helical content and a lower polarity of the protein microenvironment.
\end{abstract}

() 2011 Federation of European Biochemical Societies. Published by Elsevier B.V. All rights reserved.

\section{Introduction}

Parkinson's disease is a neurodegenerative disorder involving the functional loss of dopaminergic neurons in the substantia nigra of the midbrain [1]. The precise causes of Parkinson's disease are unknown but it is characterized by the formation of Lewy bodies, intraneuronal protein deposits rich in amyloid fibrils of $\alpha$-synuclein (AS). AS is protein of 140 amino acids expressed presynaptically, which lacks a defined secondary structure in free solution or as yet well-defined physiological functions, although it has been implicated with vesicle trafficking [2] and fatty acid metabolism [3].

AS binds to natural and artificial membranes forming an extended $\alpha$-helix oriented parallel to the membrane surface [4]. It

\footnotetext{
Abbreviations: AS, $\alpha$-synuclein; ESIPT, excited state intramolecular proton transfer; $\mathrm{L}_{\mathrm{d}}$, liquid disordered; LPR, lipid to protein ratio; MFE, $\mathrm{N}$-(2-[4-(diethylamino)phenyl]-3-hydroxy-4-oxo-4H-chromen-6-yl)-4-(2,5-dioxo-2,5-dihydro- $1 H$ pyrrol-1-yl)propanamide; MFC, 2-(2-furyl)-3-hydroxy-4-oxo-4H-chromen-6-yl)-4(2,5-dioxo-2,5-dihydro-1H-pyrrol-1-yl)propanamide; MTSL, S-(2,2,5,5-tetramethyl2,5-dihydro-1H-pyrrol-3-yl)methyl methanesulfonothioate; TEMPO, (2,2,6,6-tetramethylpiperidin-1-yl)oxidanyl

* Corresponding author. Fax: +49551201 1467.

E-mail addresses: vshvadc@gwdg.de (V.V. Shvadchak), dyushch@gwdg.de (D.A Yushchenko), rpievo@gwdg.de (R. Pievo), tjovin@gwdg.de (T.M. Jovin).

${ }^{1}$ Present address: Molecular Biosensor and Imaging Center, Carnegie Mellon University, 4400 Fifth Avenue, Pittsburgh, PA 15213, USA.
}

encompasses the N-terminal and NAC (Non-Abeta Component) regions (residues 9-90) and has an atypical periodicity (3 turns per 11 residues) [4] (Fig. 1). Depending on the system the $\alpha$-helix adopts linear [4-6] or horseshoe-like conformation [7-9]. The affinity for negatively charged bilayers is much stronger than for neutral bilayers [10] but also depends on membrane phase and curvature [11].

The N-terminal part of AS plays the determining role in membrane binding [12]. The immersion of different AS domains into the bilayer has been studied previously by EPR [4,13], tryptophan fluorescence [14,15] and NMR [16]. The NAC and N-terminal regions are fairly equally immersed in the bilayer [4] but at low lipid-to-protein ratio (LPR) the binding of the NAC region decreases [16]. The C-terminal part is flexible and is not immersed into negatively charged bilayers [13].

High resolution structural techniques require concentrated protein solutions and do not allow studies of AS orientation at the high LPRs required to ensure complete AS binding to membranes with a close to natural composition. In addition, the membranes of organelles differ in lipid composition, such that an understanding of the influences of the membrane properties and of the LPR on AS binding, conformation, and, ultimately, function, is essential.

In this work we studied the dependence of AS orientation on membrane properties and the LPR. We characterized the environment of the protein domains upon binding to artificial membranes of different composition using several independent approaches: 
(a)

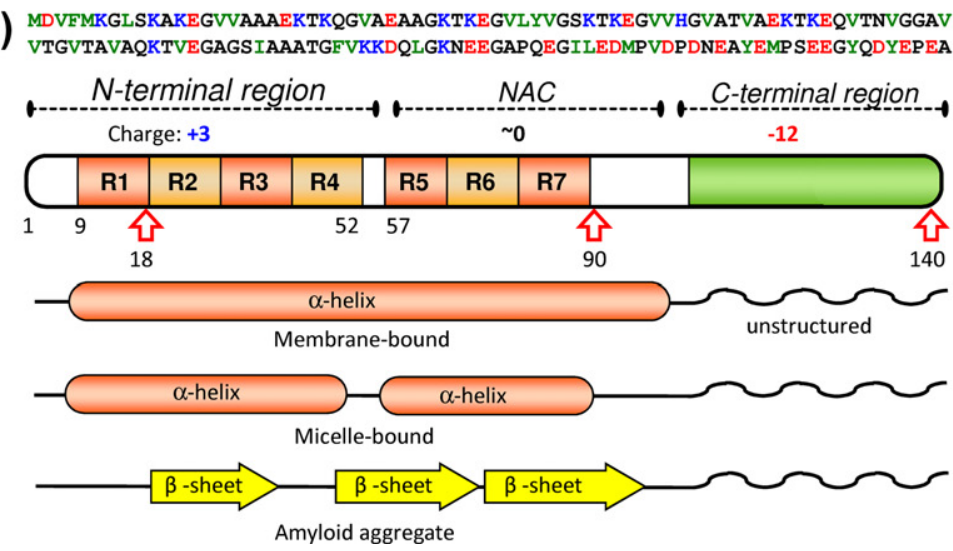<smiles></smiles>

MFC<smiles>CC1(C)C=C(CSS(C)(=O)=O)C(C)(C)N1O</smiles>

MTSL

Fig. 1. Structure of AS and labels. Labeling positions are marked by arrows. Secondary structures are based on [10].

(1) The environment polarity in the proximity of the peptide backbone was assessed by spectral changes of the solvatochromic ESIPT (Excited State Intramolecular Proton Transfer) MFC probe $[17,18]$; (2) The rigidity of the same sites was reported by EPR signal broadening of the MTSL [19]; and (3) The existence of an apolar compartment accessible for transient AS binding was assessed by the hydrophobic ESIPT probe MFE [11,20], bound through a $\sim 1 \mathrm{~nm}$ flexible linker (Fig. 1b).

The labels were placed in one of the three AS domains: $\mathrm{N}$-terminal, NAC, or C-terminal regions by labeling individual Ala-to-Cys mutants at positions 18, 90 and 140 (Fig. 1). The different environments of the AS domains sensed by the fluorescent probes revealed a decreased binding of the NAC region at high protein density and a participation of the AS C-terminus in the interaction with neutral membranes.

\section{Materials and methods}

All experiments with AS were performed in $25 \mathrm{mM} \mathrm{Na}-\mathrm{PO}_{4}, \mathrm{pH}$ 6.5, $150 \mathrm{mM} \mathrm{NaCl}$ buffer. Fluorescence and CD spectra were recorded at $37^{\circ} \mathrm{C}$.

\subsection{Circular dichroism}

Circular dichroism spectra were recorded with a JASCO-J720 spectropolarimeter. Protein and lipid concentrations were $4 \mu \mathrm{M}$
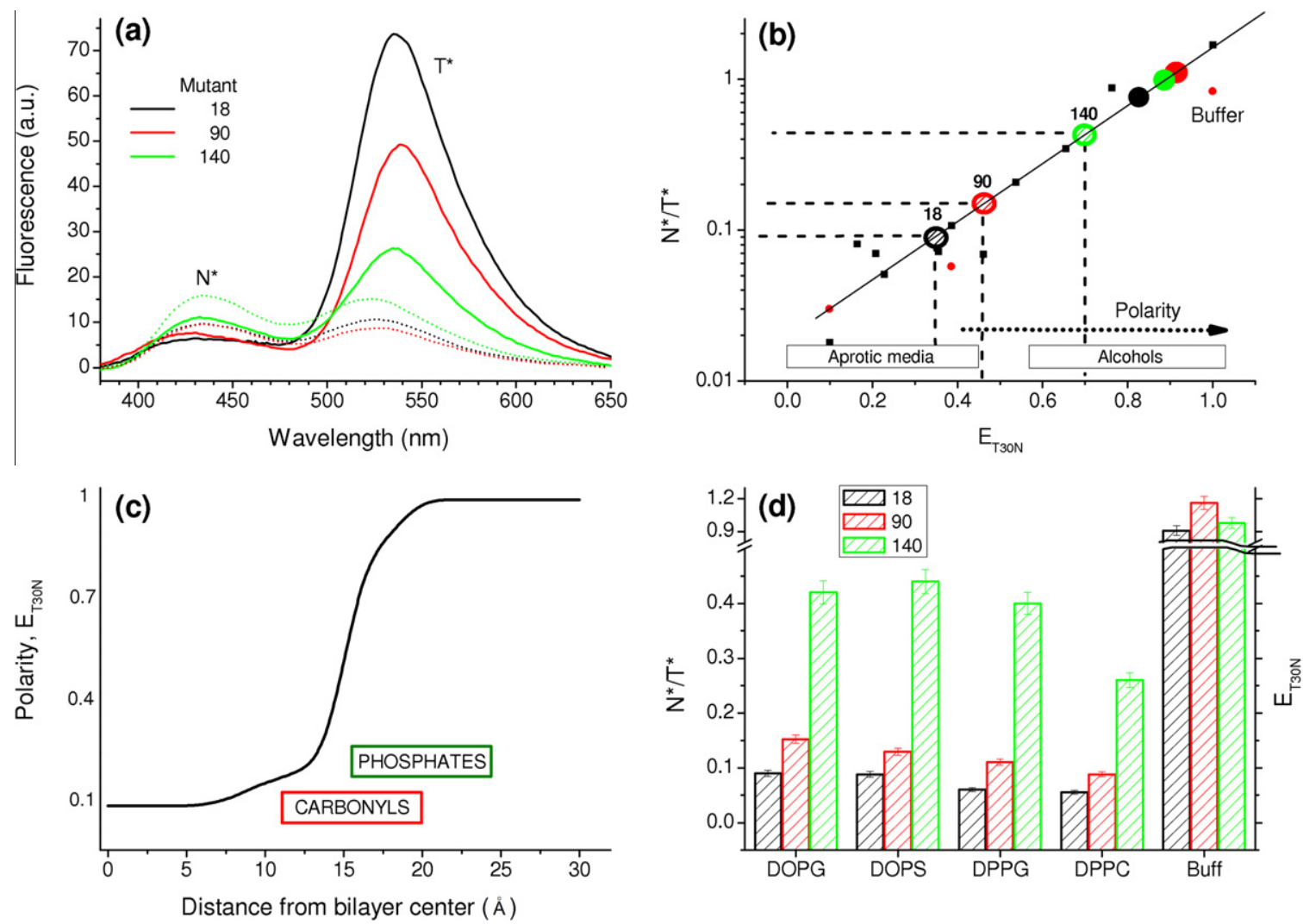

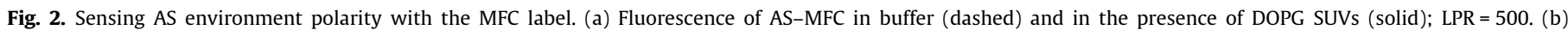

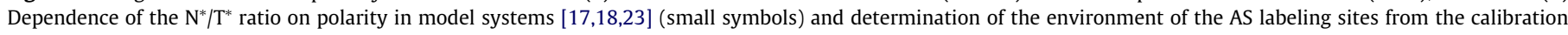

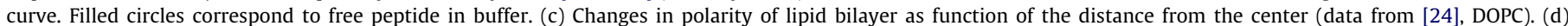

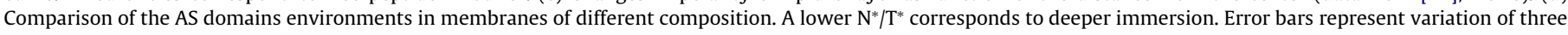
experiments. AS mutants with label at positions 18, 90 and 140 are marked on all panels by black, red and green, respectively. 
and $1 \mathrm{mM}$ respectively. The calculation of helicity was performed as described previously [21].

\subsection{Fluorescence spectra}

Emission spectra were recorded with a Cary Eclipse spectrofluorimeter (Varian). The protein concentration was $100-400 \mathrm{nM}$. Lipids were in 500-fold excess, insuring $>97 \%$ protein binding [11]. The excitation wavelengths for MFE and MFC were 410 and $350 \mathrm{~nm}$, respectively. The $\mathrm{N}^{*} / \mathrm{T}^{*}$ emission band ratios were calculated from the peak intensities and showed a $<5 \%$ (relative) variation in three independent determinations.

In quenching experiments, solutions of AS labeled by MFE in presence of excess (500 lipids per protein) of SUV were titrated with a $100 \mathrm{mM}$ solution of TEMPO ((2,2,6,6-tetramethylpiperidin1-yl)oxidanyl) (neutral stable radical) in DMSO. The quenching constants were determined by fitting the intensity plots to $I / I_{0}=1+K_{\mathrm{q}} \cdot \mathrm{C}_{\mathrm{TEMPO}}$.

\subsection{EPR}

EPR spectroscopic measurements were carried out on a Bruker Elexsys E500 CW-EPR equipped with a Super-X microwave bridge operating at 9.3-9.5 GHz and a standard Bruker X-band ER4119SHQE cavity. Sample solution spectra were acquired at room temperature $\left(23 \pm 2{ }^{\circ} \mathrm{C}\right)$ in a quartz $150 \mu \mathrm{L}$-flat cell (ER160FC-Q) using $5 \mathrm{~mW}$ incident microwave power, $3.5 \mathrm{G}$ field modulation amplitude at $100 \mathrm{kHz}, 150$ Gauss sweep width. Lipid and protein concentrations were $5 \mathrm{mM}$ and $20 \mu \mathrm{M}$ respectively.

\subsection{Protein preparation, purification and labeling}

AS and its single cysteine mutants were expressed and purified as described earlier [11].

(a)

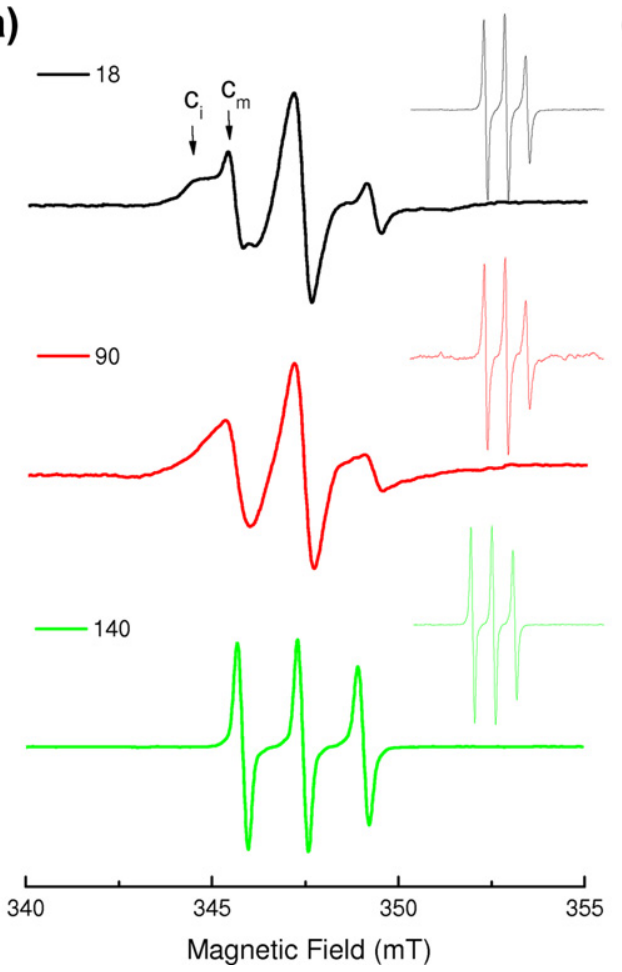

The MFC and MFE labels were synthesized as described in literature $[11,18]$. MTSL was purchased from Sigma-Aldrich.

\subsubsection{AS-MFE and AS-MFC}

AS cysteine mutants were labeled by adding 2-15-fold excess of MFC or MFE solution in DMSO ( $48 \mathrm{~h}$ at $4{ }^{\circ} \mathrm{C}$, pH 7.2 buffer, $5 \mathrm{mM}$ TCEP) and purified by exclusion chromatography using Superdex 200HR 10/30 SEC column (See SI). Recovery 70\%. The labeling efficiency, $>90 \%$, was determined from the absorbance of the dye (FE $\varepsilon_{430 \mathrm{~nm}}=32,000 \mathrm{M}^{-1} \mathrm{~cm}^{-1}, \quad \varepsilon_{275 \mathrm{~nm}}=14,100 \mathrm{M}^{-1} \mathrm{~cm}^{-1} ; \quad \mathrm{FC}$ $\varepsilon_{345 \mathrm{~nm}}=16,000 \mathrm{M}^{-1} \mathrm{~cm}^{-1} \varepsilon_{275 \mathrm{~nm}}=4800 \mathrm{M}^{-1} \mathrm{~cm}^{-1}$ ) and the protein $\left(\varepsilon_{275 \mathrm{~nm}}=5600 \mathrm{M}^{-1} \mathrm{~cm}^{-1}\right)$.

\subsubsection{AS-MTSL}

To $1 \mathrm{ml}$ of a $0.2 \mathrm{mM}$ solution of single cysteine mutant of AS in phosphate buffer, pH 7.2, $5 \mathrm{mM}$ TCEP, $0.25 \mathrm{mg}$ ( $\sim$-fold excess) of MTSL ( $S$-(2,2,5,5-tetramethyl-2,5-dihydro-1H-pyrrol-3-yl)methyl methanesulfonothioate) label in $0.1 \mathrm{ml}$ of DMSO was added. The reaction was left for $48 \mathrm{~h}$ at $4{ }^{\circ} \mathrm{C}$ with mixing. The excess label, TCEP, and DMSO were removed by gel filtration using PD10 columns (GE Healthcare). The estimated labeling efficiency was $>90 \%$.

\subsection{Preparation of SUVs}

SUVs were prepared by evaporation of $\mathrm{CHCl}_{3}$ lipid solutions, hydration of the resulting films in buffer and $1 \mathrm{~h}$ sonication at temperatures $5-15^{\circ} \mathrm{C}$ above the transition points. The size of all vesicles and their stability in the presence of protein were checked by DLS. (See SI for details.)

\section{Results and discussion}

The orientation of AS in membranes was determined as a function of the composition and LPR by measuring polarity, rigidity and

(b)
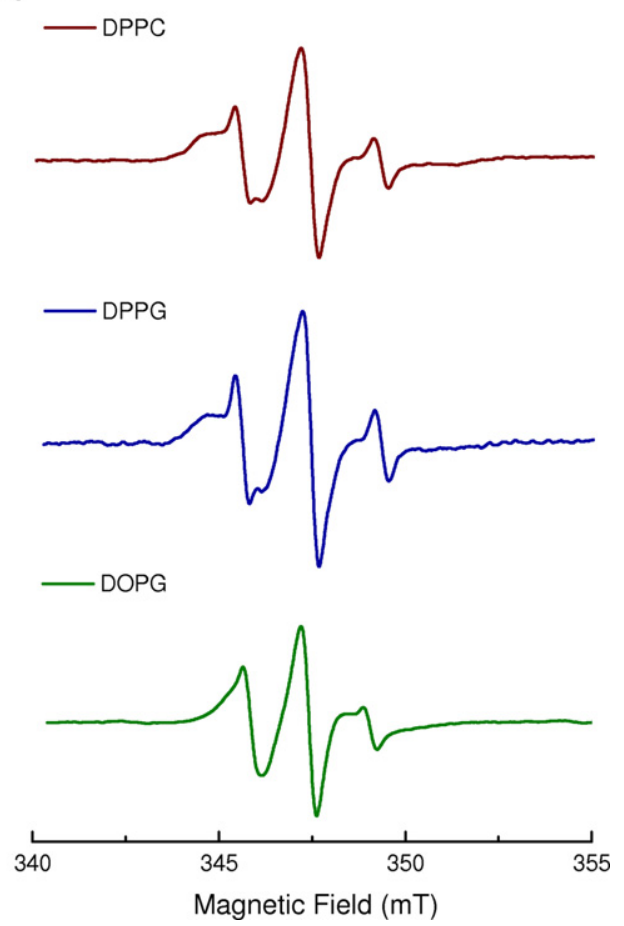

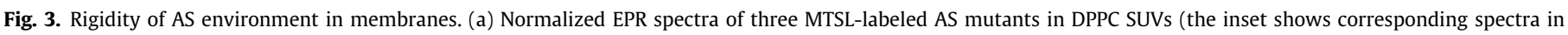

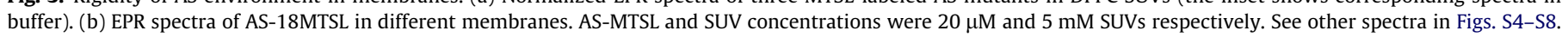


Table 1

Changes of AS environment upon binding to membrane

\begin{tabular}{|c|c|c|c|c|c|c|c|}
\hline \multirow[b]{2}{*}{ System } & \multicolumn{2}{|l|}{ Label } & \multicolumn{2}{|l|}{ MFC } & \multirow{2}{*}{$\begin{array}{l}\text { MTSL } \\
\Delta \mathrm{H}_{0}^{-1} \mathrm{G}^{-1}\end{array}$} & \multicolumn{2}{|c|}{ MFE } \\
\hline & Label position & $\mathrm{N}^{*} / \mathrm{T}^{*}$ & Polarity, $\mathrm{E}_{\mathrm{T} 30 \mathrm{~N}}$ & $\mathrm{I} / \mathrm{I}_{0}$ & & QY \% & $\mathrm{K}_{\mathrm{q}} \mathrm{M}^{-1}$ \\
\hline \multirow{3}{*}{ Buffer } & 18 & 0.91 & $>0.9$ & 1 & 0.34 & 5.0 & 400 \\
\hline & 90 & 1.46 & $>0.9$ & 1 & 0.34 & 6.2 & 400 \\
\hline & 140 & 0.98 & $>0.9$ & 1 & 0.34 & 4.7 & 450 \\
\hline DOPG & 18 & 0.09 & 0.35 & 4.9 & 0.24 & 32 & 50 \\
\hline$(-1)$ & 90 & 0.15 & 0.45 & 3.6 & 0.24 & 32 & 45 \\
\hline$L_{d}$ & 140 & 0.42 & 0.70 & 1.4 & 0.34 & 33 & 350 \\
\hline DPPC & 18 & 0.055 & 0.29 & 5.3 & 0.24 & 34 & 140 \\
\hline (0) & 90 & 0.088 & 0.34 & 4.0 & 0.22 & 30 & 130 \\
\hline Gel & 140 & 0.26 & 0.56 & 2.2 & 0.34 & 30 & 145 \\
\hline DPPG & 18 & 0.06 & 0.30 & 5.6 & 0.27 & 35 & 47 \\
\hline$(-1)$ & 90 & 0.11 & 0.39 & 3.2 & 0.24 & 41 & 53 \\
\hline $\mathrm{Gel}$ & 140 & 0.40 & 0.68 & 1.4 & 0.34 & 31 & 140 \\
\hline
\end{tabular}

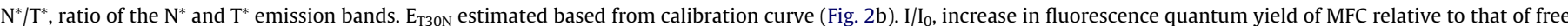

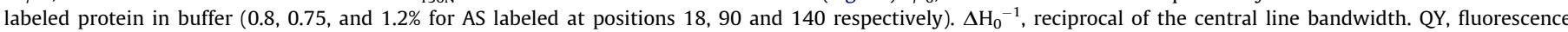

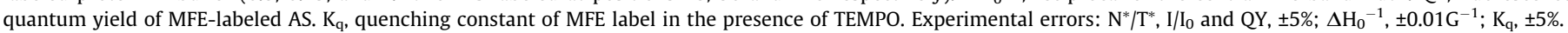

water-accessibility of the three proteins domains. Single cysteine mutants of AS at positions 18, 90 and 140 were labeled with the (1) polarity-sensitive MFC probe, (2) the rigidity-sensitive MTSL spin-label, and (3) the MFE probe highly sensitive to membrane proximity. The binding of all labeled proteins to membrane(s) was compared to that of WT AS using CD spectroscopy, and showed $<10 \%$ differences in binding stoichiometry (Fig. S9). We compared the responses of the labels upon binding under conditions of excess lipid (bilayer) and employed the most sensitive (MFE) probe to sense the dependence of AS conformation and orientation in the bound state on the LPR.

\subsection{Polarity of labeling sites}

For estimating the environment polarities of the AS domains we applied MFC, a solvatochromic label based on a small water-soluble dye. This label was previously used for monitoring AS aggregation and does not significantly modify the fibrilization kinetics [18]. MFC reports the polarity and solvation ability ( $\left.E_{\mathrm{T} 30 \mathrm{~N}}\right)$ of the environment by the ratio of the dual emission bands corresponding to the normal $\left(\mathrm{N}^{*}, \sim 430 \mathrm{~nm}\right)$ and tautomeric $\left(\mathrm{T}^{*}, \sim 520 \mathrm{~nm}\right)$ excited state species [22]. A decrease in polarity leads to a significant increase in the $T^{*}$ band and thus a diminution of the $\mathrm{N}^{*} / \mathrm{T}^{*}$ emission band ratio [23].

In the case of free monomeric AS in buffer, MFC reported an environment typical for unstructured proteins and peptides [1718 ]. Upon addition of each of the AS-MFC conjugates to an excess of lipid vesicles, the probe indicated a decrease in polarity (Fig. 2a, Table 1$)$. The emission band ratio $\left(\mathrm{N}^{*} / \mathrm{T}^{*}\right)$ of MFC depends on solvent polarity $\left(\mathrm{E}_{\mathrm{T} 30 \mathrm{~N}}\right)$. We established a calibration curve based on data for the probe in model organic solvents [17,23] (Fig. 2b) and estimated the polarity of MFC attached to different AS domains. The values were then compared with the calculated profile of polarity as function of the distance from the bilayer center [24]. The environments of the labels at positions 18 and 90 were similar to those of polar aprotic media, suggesting an immersion of the protein only to the level of the lipid polar heads. The environment of the C-terminus was much more polar but notably different from that of bulk water (Fig. 2), corresponding rather to the hydration shell of lipid phosphate groups [24].

The environment of AS bound to membranes in the gel phase (DPPC, DPPG) was characterized by a lower polarity than in the case of the $\mathrm{L}_{\mathrm{d}}$ (liquid disordered) phase (Table $1, \mathrm{~N}^{*} / \mathrm{T}^{*}$ values for positions 18 and 90), indicating a deeper insertion of the protein into the bilayer. The lowest polarity (deepest immersion) of the C-terminus was observed in the presence of rigid neutral DPPC (Fig. 2d, Table 1) probably due to the absence of electrostatic repulsion.

\subsection{Rigidity}

The rigidity of the MTSL label conjugated to AS in the membranes was probed using EPR spectroscopy. The spin-label was attached to the same positions as MFC. The EPR spectra of all three
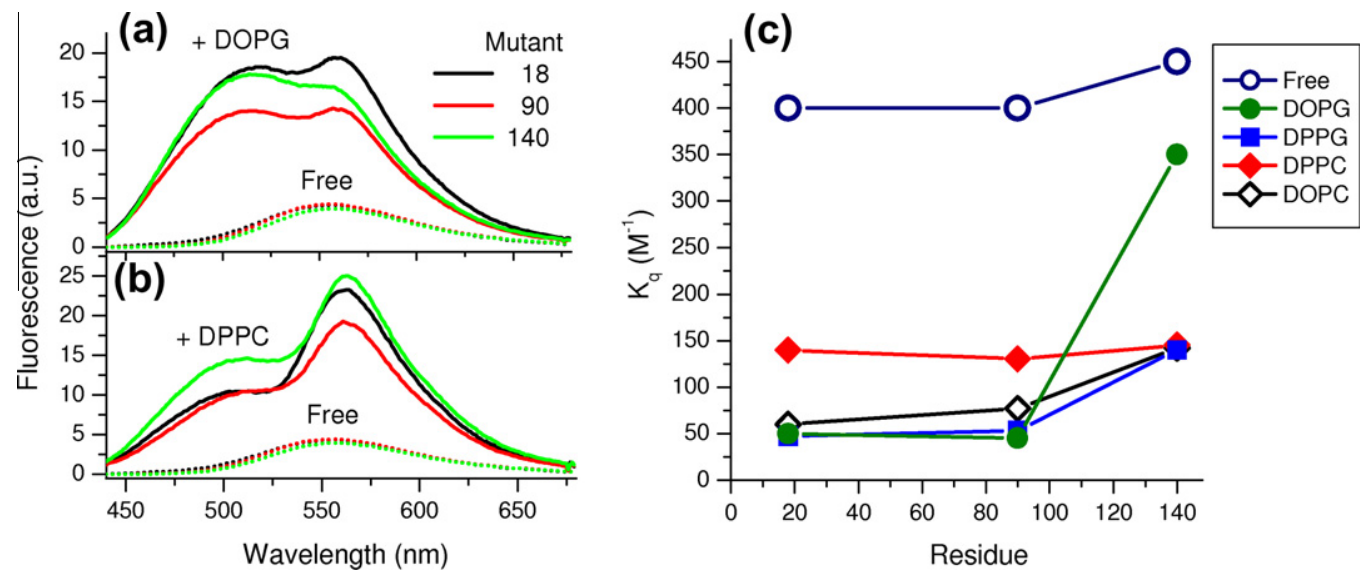

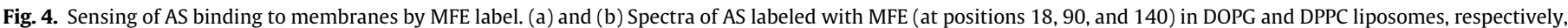

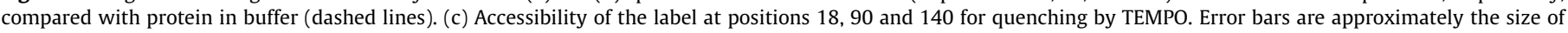
symbols. See titration curves in Fig. S8. 
AS mutants free in solution showed very sharp lines (reciprocal of the central line bandwidth: $\Delta H_{0}^{-1}=0.34 \mathrm{G}^{-1}$ ) consistent with a high degree of rotational freedom typical for unstructured protein domains (Fig. 3a, inset) [25]. Upon membrane binding, the EPR spectra of AS mutants labeled at sites 18 or 90 but not 140 showed broadening and a decreased intensity of the external hyperfine lines, indicative of a rigid medium (Fig. 3a) [26]. The broadening of the spectra was consistent with a shallow location of the probe on/in the membrane, with the AS $\alpha$-helix oriented parallel to the water/membrane interface, as shown for anionic membranes in the $L_{d}$ phase $[4,13]$. The probe at position 18 in neutral (DPPC) and anionic (DPPG) membranes in the gel phase indicated greater rigidity than in the case of anionic disordered phase bilayers (DOPG) (Fig. 3b), although the degree of immobilization was much less than that typically observed at buried sites in well packed membrane proteins [26].

In some cases the EPR spectra were consistent with the presence of more than one component. In particular, the EPR spectra of AS-18MTSL showed the superposition of at least two components, $C_{m}$ and $C_{i}$, with $C_{m}$ corresponding to the spectrum of a species with high mobility (sharp lines), and $C_{i}$ to a less mobile spin population (broadened components). $C_{m}$ may represent free AS in solution, or AS bound to the membrane, except for the labeled region with the label $[4,13]$. Inasmuch as the high LPR $(250)$ insured $>95 \%$ protein binding, one can assume that the main contribution to $C_{m}$ was from the bound protein.

\subsection{Water accessibility}

We coupled MFE, a fluorescent probe highly sensitive to environment polarity and hydration, to AS cysteine mutants through a $\sim 1 \mathrm{~nm}$ flexible linker. The dye is relatively lipophilic and therefore senses the minimal polarity in close proximity to the labeling site rather than the mean environment polarity sensed by MFC. MFE signals binding of AS to model membranes by an increase in the fluorescence quantum yield and the appearance of the twoband emission (Fig. 4) characteristic for an aprotic environment [11]. The ratio of the two emission bands $\left(\mathrm{N}^{*} / \mathrm{T}^{*}\right)$ depends on the relative immersion of the label into a lipid bilayer $[20,22]$ and has been used as a signature of AS bound to membranes of defined composition [11].

Binding of MFE-labeled AS to membranes in the gel phase (DPPC and DPPG) resulted in a lower emission band ratio than in the case of disordered bilayers (Fig. 4), pointing to a deeper immersion of the AS N-terminal and NAC regions, in accordance with the responses of MTSL and MFC labels. All of the probes indicated a lack of correlation between the level of immersion of the AS N-terminal and NAC regions in the bilayer, and the affinity for the membrane [11].

Interestingly, MFE at residue 140 also indicated proximity to the bilayer, even though the MFC and MTSL probes with shorter linkers showed only minor changes in polarity and rigidity for this position. We conclude that the C-terminus was situated at a distance less than $1 \mathrm{~nm}$ from the lipophilic bilayer and was also perturbed by the interaction.

A more precise determination of the position of the MFE label in the membrane and its accessibility to water was achieved in a quenching experiment using a neutral quencher TEMPO (Table 1 , Fig. 4c). In free solution, the quenching of the label was strong for all three labeling sites, as expected for an unstructured protein. Binding to rigid DPPC and DPPG SUVs led to significant screening of all positions (Fig. 4c). In contrast, in negatively charged disordered DOPG the quenching of the label at C-terminus was modified only slightly, whereas the two other segments were efficiently shielded from water. This observation can be interpreted in the context of an interaction of the C-terminus with membranes via a

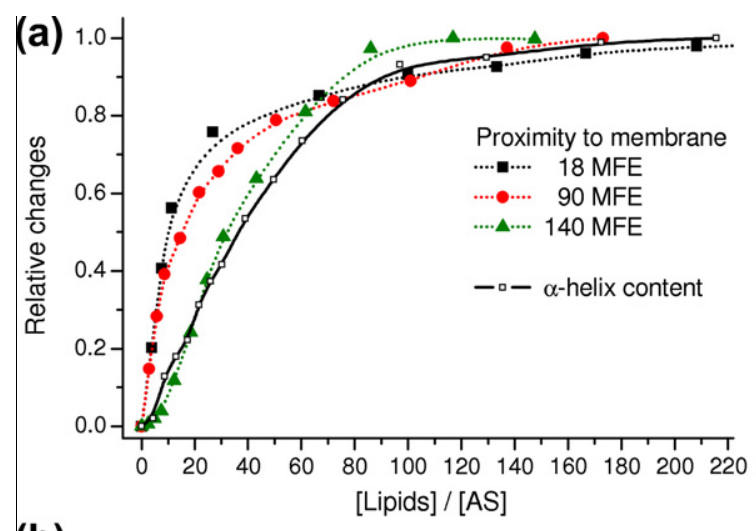

(b)
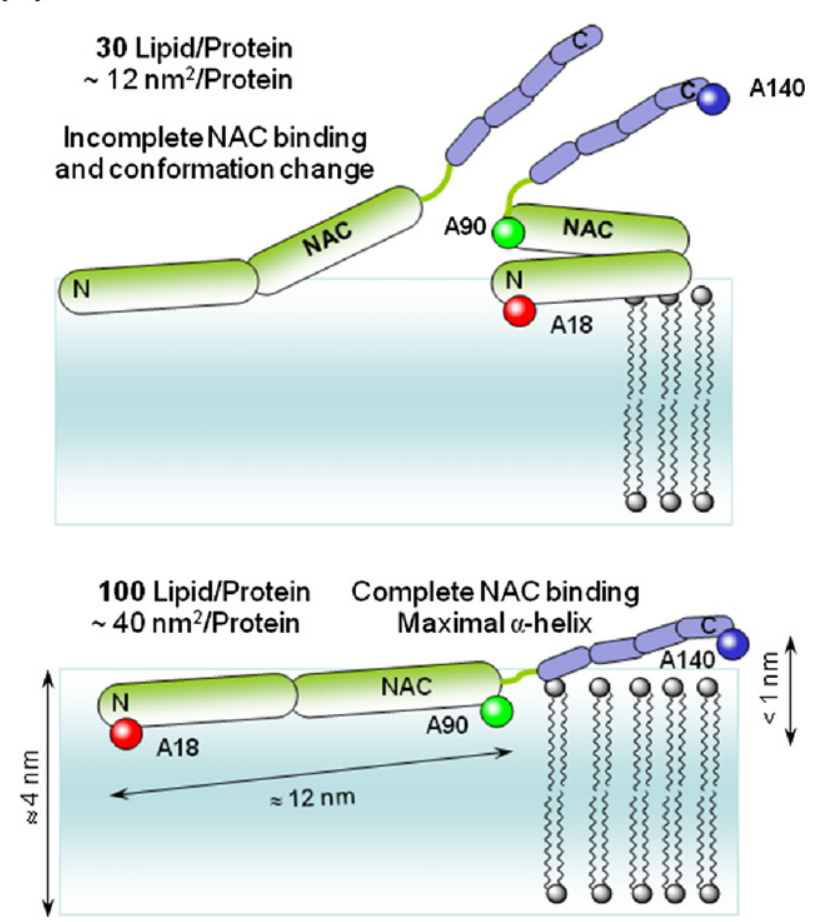

Fig. 5. Dependence of AS conformation on LPR. (a) Titration of AS by DOPG/DOPC $(1: 1)$ vesicles. Relative changes of fluorescence intensities of AS labeled by MFE at positions 18 (black), 90 (red) and 140 (green) compared with degree of $\alpha$-helix formation ( $C D$ at $222 \mathrm{~nm}$, open squares). (b) Scheme of AS orientation on membrane at low (top) and high (bottom) LPRs.

charge-independent mechanism that is probably mediated by membrane defects $[11,27]$.

\subsection{Dependence of the orientation on the AS to lipid ratio}

We studied the dependence of the orientation of AS on/in membranes on the LPR by comparing the responses of the labels at the different positions in the protein sequence upon titration with liposomes. In most cases, the binding curves constructed from the response of the label at the $\mathrm{N}$-terminus did not coincide with the calculation of the $\alpha$-helical content measured by CD. For example, the fluorescence of AS-18MFE showed that binding to DOPG/DOPC vesicles was characterized by a stoichiometry of $\sim 20$ lipids per protein, whereas the $\mathrm{CD}$ measurements showed that the maximal transition of the protein to $\alpha$-helical conformation occurred at $\sim 80$ lipids per AS (Fig. 5a). At a ratio of 30 lipids per AS the N-terminus and the NAC region were almost completely membrane bound, yet the label at the C-terminus showed only $<50 \%$ of the 
maximal fluorescence increase (Fig. 5a). This LPR corresponds to a very dense membrane surface coverage by AS ( $\sim 12 \mathrm{~nm}^{2} /$ protein), a value even smaller than the size of a 90 aa $\alpha$-helical fragment $(\sim 14 \times 1 \mathrm{~nm}), \alpha$-Helix formation under this condition was $<50 \%$ of the maximal value. Thus, when the protein is in excess, it binds via the $\mathrm{N}$-terminal domain that has the highest affinity, leaving the C-terminus completely water exposed because of the lack of accessible membrane surface (Figs. S2 and S3). The relatively low degree of the transition to the $\alpha$-helical form at these low LPRs is probably due to incomplete binding of the NAC region, although position 90 was clearly close to the membrane according to the fluorescent probe. At higher LPRs $(>100)$ the membrane surface is sufficient for binding of all AS domains (>40 $\mathrm{nm}^{2} /$ protein) and both the $\mathrm{N}$ terminal and NAC regions assume an $\alpha$-helical conformation and are fully bound. Moreover, the bilayer surface is also accessible to the C-terminus and it participates in the interaction with the membrane (Fig. 5b).

The difference in AS/membrane binding stoichiometry determined by CD and fluorescence of the MFE label placed at the N-terminus indicates that the size of $\alpha$-helical domains of the protein in membrane bound form depends on the LPR and increases in the presence of excess lipid. The membrane composition can also impact the conformation of AS [12]. Indeed, we found that in rigid DPPG SUVs, AS has a $262 \% \alpha$-helical content, yet only $50 \%$ in charged membranes in the $L_{d}$ phase (DOPG) (Table S1). This result is particularly interesting since the affinity of AS for DPPG is lower than for DOPG [11]. Thus, binding affinity is not directly correlated with the extent of $\alpha$-helix formation.

We observed differences in the binding profiles of the $\mathrm{N}$ - and $\mathrm{C}$ terminal segments of AS in many charged model membranes (Figs. S1, S2 and S3). The binding of AS to neutral membranes is characterized by virtually identical titration curves of the three labeled mutants. This difference points to a significant role of electrostatic interactions between the membrane surface and the protein N-terminus in the dense mode of AS binding and to a more lipophilic nature of the interaction in the more stable conformation adopted by AS in the presence of excess lipid.

Different modes of AS binding to negatively charged multilamellar vesicles depending on the LPR were observed recently by NMR [16]. As in the case of our study, it was shown that the relative involvement of the N-terminal segment of AS in membrane binding increases at low LPRs. The fact that the N-terminus of AS has a strong affinity for negatively charged membranes and can mediate very dense membrane binding is also supported by the observation that truncation of first 6 amino acids strongly decreases the affinity of AS for membranes [12].

\section{Conclusions}

In summary, we conclude that binding of AS to negatively charged membrane does not necessarily require a complete transition to $\alpha$-helical form, but rather can be mediated by the N-terminus alone with a requirement for minimal membrane surface $\left(<15 \mathrm{~nm}^{2} /\right.$ molecule). In the presence of lipid excess there are no significant changes in AS orientation and immersion upon alterations in membrane phase and charge. Upon binding of AS to the model membranes the $\mathrm{N}$-terminus and NAC regions adopt $\alpha$-helical conformation [10]. The labels at positions 18 and 90 indicate greater rigidity (MTSL) and shielding from water (MFC, MFE), in accordance with previous results and a model with water/membrane interfacial orientation of AS $\alpha$-helix [4]. However, it remains to be established whether the $\alpha$-helical conformation precedes, accompanies, or is subsequent to binding. Access to fast kinetic techniques and the use of fluorescent $\alpha$-helix reporters should resolve this fundamental question.

\section{Acknowledgments}

This study was founded by Toxic Proteins Conformation project of the Max Planck Society and by Marie Curie Actions postdoctoral fellowships to VVS and DAY. We thank Irina Karyagina for initiating this project, Marina Bennati for access to the EPR spectrometer and discussions, and Lisandro Falomir-Lockhart for valuable comments.

\section{Appendix A. Supplementary data}

Supplementary data associated with this article can be found, in the online version, at doi:10.1016/j.febslet.2011.10.006.

\section{References}

[1] Lees, A.J., Hardy, J. and Revesz, T. (2009) Parkinson's disease. Lancet 373, 2055 2066.

[2] Nemani, V.M. et al. (2010) Increased expression of $\alpha$-synuclein reduces neurotransmitter release by inhibiting synaptic vesicle reclustering after endocytosis. Neuron 65, 66-79.

[3] Golovko, M.Y., Rosenberger, T.A., Feddersen, S., Faergeman, N.J. and Murphy, E.J. (2007) Alpha-synuclein gene ablation increases docosahexaenoic acid incorporation and turnover in brain phospholipids. J. Neurochem. 101, 201-211.

[4] Jao, C.C., Hegde, B.G., Chen, J., Haworth, I.S. and Langen, R. (2008) Structure of membrane-bound alpha-synuclein from site-directed spin labeling and computational refinement. Proc. Natl. Acad. Sci. USA 105, 19666-19671.

[5] Georgieva, E.R., Ramlall, T.F., Borbat, P.P., Freed, J.H. and Eliezer, D. (2008) Membrane-bound alpha-synuclein forms an extended helix: long-distance pulsed ESR measurements using vesicles, bicelles, and rodlike micelles. J. Am. Chem. Soc. 130, 12856-12857.

[6] Trexler, A.J. and Rhoades, E. (2009) $\alpha$-Synuclein binds large unilamellar vesicles as an extended helix. Biochemistry 48, 2304-2306.

[7] Lokappa, S.B. and Ulmer, T.S. (2011) $\alpha$-Synuclein populates both elongated and broken helix states on small unilamellar vesicles. J. Biol. Chem. 286, 2145021457.

[8] Robotta, M., Braun, P., van Rooijen, B., Subramaniam, V., Huber, M. and Drescher, M. (2011) Direct evidence of coexisting horseshoe and extended helix conformations of membrane-bound alpha-synuclein. ChemPhysChem 12, 267-269.

[9] Rao, J.N., Jao, C.C., Hegde, B.G., Langen, R. and Ulmer, T.S. (2010) A combinatorial NMR and EPR approach for evaluating the structural ensemble of partially folded proteins. J. Am. Chem. Soc. 132, 8657-8668.

[10] Bisaglia, M., Mammi, S. and Bubacco, L. (2009) Structural insights on physiological functions and pathological effects of $\alpha$-synuclein. FASEB J. 23, 329-340.

[11] Shvadchak, V.V., Falomir-Lockhart, L.J., Yushchenko, D.A. and Jovin, T.M. (2011) Specificity and kinetics of $\alpha$-synuclein binding to model membranes determined with fluorescent Excited State Intramolecular Proton Transfer (ESIPT) Probe. J. Biol. Chem. 286, 13023-13032.

[12] Bartels, T., Ahlstrom, L.S., Leftin, A., Kamp, F., Haass, C., Brown, M.F. and Beyer, K. (2010) The N-terminus of the intrinsically disordered protein alpha-synuclein triggers membrane binding and helix folding. Biophys. J 99, 2116-2124.

[13] Drescher, M., Godschalk, F., Veldhuis, G., van Rooijen, B.D., Subramaniam, V. and Huber, M. (2008) Spin-label EPR on alpha-synuclein reveals differences in the membrane binding affinity of the two antiparallel helices. ChemBioChem 9, 2411-2416

[14] Pfefferkorn, C.M. and Lee, J.C. (2010) Tryptophan probes at the $\alpha$-synuclein and membrane interface. J. Phys. Chem. B 114, 4615-4622.

[15] van Rooijen, B.D., van Leijenhorst-Groener, K.A., Claessens, M.M. and Subramaniam, V. (2009) Tryptophan fluorescence reveals structural features of alpha-synuclein oligomers. J. Mol. Biol. 394, 826-833.

[16] Bodner, C.R., Dobson, C.M. and Bax, A. (2009) Multiple tight phospholipidbinding modes of alpha-synuclein revealed by solution NMR spectroscopy. J. Mol. Biol. 390, 775-790.

[17] Shvadchak, V.V., Klymchenko, A.S., de Rocquigny, H. and Mely, Y. (2009) Sensing peptide-oligonucleotide interactions by a two-color fluorescence label: application to the HIV-1 nucleocapsid protein. Nucl. Acids Res. 37, e25.

[18] Yushchenko, D.A., Fauerbach, J.A., Thirunavukkuarasu, S., Jares-Erijman, E.A and Jovin, T.M. (2010) Fluorescent ratiometric MFC probe sensitive to early stages of alpha-synuclein aggregation. J. Am. Chem. Soc. 132, 7860-7861.

[19] Gordon-Grossman, M., Gofman, Y., Zimmermann, H., Frydman, V., Shai, Y., Ben-Tal, N. and Goldfarb, D. (2009) A combined pulse EPR and Monte Carlo simulation study provides molecular insight on peptide-membrane interactions. J. Phys. Chem. B 113, 12687-12695.

[20] Postupalenko, V.Y., Shvadchak, V.V., Duportail, G., Pivovarenko, V.G. Klymchenko, A.S. and Mely, Y. (2011) Monitoring membrane binding and insertion of peptides by two-color fluorescent label. Biochim. Biophys. Acta 1808, 424-432.

[21] Scholtz, J.M., Qian, H., York, E.J., Stewart, J.M. and Baldwin, R.L. (1991) Parameters of helix-coil transition theory for alanine-based peptides of varying chain lengths in water. Biopolymers 31, 1463-1470. 
[22] Demchenko, A.P., Mely, Y., Duportail, G. and Klymchenko, A.S. (2009) Monitoring biophysical properties of lipid membranes by environmentsensitive fluorescent probes. Biophys. J. 96, 3461-3470.

[23] Klymchenko, A.S. and Demchenko, A.P. (2004) 3-Hydroxychromone dyes exhibiting excited-state intramolecular proton transfer in water with efficien two-band fluorescence. New J. Chem. 28, 687-692.

[24] Lomize, A.L., Pogozheva, I.D. and Mosberg, H.I. (2011) Anisotropic solvent model of the lipid bilayer. 2. Energetics of insertion of small molecules, peptides, and proteins in membranes. J. Chem. Inf. Model 51, 930-946.
[25] McHaourab, H.S., Lietzow, M.A., Hideg, K. and Hubbell, W.L. (1996) Motion of spin-labeled side chains in T4 lysozyme, correlation with protein structure and dynamics. Biochemistry 35, 7692-7704.

[26] Bordignon, E. and Steinhoff, H.J. (2007) Membrane protein structure and dynamics studied by site-directed spin-labeling ESR. ESR Spectrosc. Membr. Biophys. 27, 129-164.

[27] Nuscher, B., Kamp, F., Mehnert, T., Odoy, S., Haass, C., Kahle, P.J. and Beyer, K. (2004) Alpha-synuclein has a high affinity for packing defects in a bilayer membrane - A thermodynamics study. J. Biol. Chem. 279, 21966-21975. 